\title{
OPTIMIZATION OF WINDOWING AND PEAK-WINDOWING TECHNIQUES FOR WCDMA SYSTEMS
}

\author{
H. Kuo and S. W. Cheung \\ Department of Electrical and Electronic Engineering \\ The University of Hong Kong, Pokfulam Road, Hong Kong \\ \{hkuo,swcheung\}@eee.hku.hk
}

\begin{abstract}
In the downlink of Wideband Code Division Multiple Access (WCDMA) system, the signals from different channels are combined together before power amplification and transmission. The combined signal has a very high crest factor (CF) which imposes a tight requirement on the linearity of the transmitter power amplifier (PA). This paper proposes a peak-windowing technique to reduce the $\mathrm{CF}$. Computer simulation results show that the proposed technique is superior to the conventional windowing technique. Since different window functions and lengths of window affect the performances of the techniques, in this paper, an attempt is also made to optimize the type of window, the length and other parameters for different windows in terms of the signal-to-noise ratio (SNR) and the bit-error-rate (BER) performance.
\end{abstract}

\section{INTRODUCTION}

In a Wideband Code Division Multiple Access (WCDMA) base station, the transmitted signal in the downlink consists of a large number of CDMA signals from different users. This leads to a very high peak-to-average power ratio (PAP), also known as the crest factor (CF) [1], in the downlink WCDMA signal and imposes a tight restriction on the linearity of the power amplifiers (PAs) used in the base stations. A highly linear PA requires a high DC power consumption, leading to low power efficiency. In order to have good power efficiency in the PA, the CF of the signal needs to be reduced. One of the ways to do this is to use different clipping and windowing techniques which have been proposed and studied [2]-[3]. This paper proposes a new peak-windowing technique to reduce the CF of the WCDMA signal. Computer simulations using Matlab and Simulink have been used to compare the effects of the proposed technique and the conventional windowing on the WCDMA signal from a base station. Results have shown that the proposed peak-windowing technique is superior to the conventional windowing technique in terms of the signal quality-metric, i.e., the error-vector-magnitude (EVM), the peak-code-domain error (PCDE) and the adjacent-channelleakage power ratio (ACLR), specified in the 3GPP specification [4], and the reduction of $\mathrm{CF}$ and the bit-errorrate (BER) performances. Since the length of the window used affects the performance of the technique, an attempt is also made in the paper to optimize the different window type, length and other parameters for in terms of the SNR and BER performance.

\section{STSTEM DESCRIPTION}

The WCDMA system model used for studies is shown in Fig. 1, which consists of 16 data channels and 3 control channels. This model is the same as the Test Model 3 in [4]. The data symbols generated for the data and control channels are random. The data symbols in each channel are spread by a prescribed spreading code with a spreading factor of 256 and scaled by a prescribed power level. The signals in all these channels are added together and then filtered by a transmitter pulse shaping filter with a frequency response of a root-raised-cosine shape having a roll-off factor of 0.22 . The signal from the filter output is sampled 4 times per chip. Clipping and windowing are then applied here if used. The envelopes of the in-phase signal, $\mathrm{I}(\mathrm{t})$, and the quaduature signal, $\mathrm{Q}(\mathrm{t})$ can be modeled as Gaussian distributed random variables with zero mean and variance $\sigma^{2}$. The clipping ratio, $\mathrm{CR}$, is defined as

$$
C R=\frac{A}{\sigma}
$$

\section{WINDOWING TECHNIQUES}

\subsection{Conventional Windowing [3]}

In the conventional windowing technique, the envelope of the complex-valued signal, $x(n)$, at the output of the pulse shaping filter of Fig. 1, are clipped at a maximum permissible amplitude $A$ to give

$$
y(n)=c(n) x(n)
$$




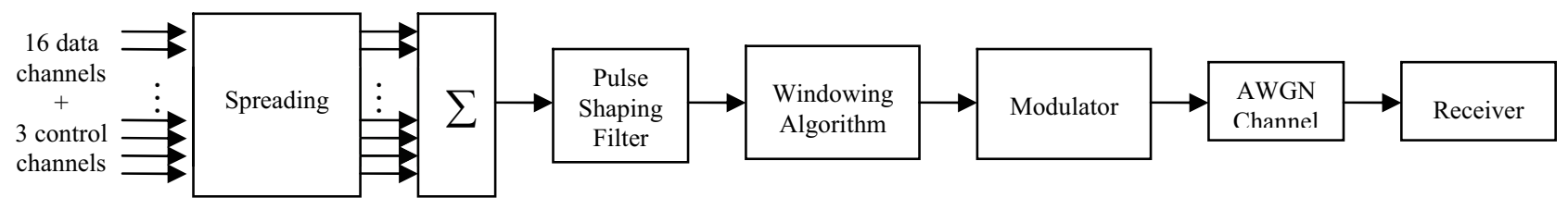

Figure 1. WCDMA system model

where $c(n)$ is the clipping function given by

$$
c(n)=\left\{\begin{array}{cc}
\frac{A}{|x(n)|}, & |x(n)|>A \\
1, & |x(n)| \leq A
\end{array}\right.
$$

Obviously, the clipping process creates sharp corners in signal envelops, causing the signal spectrum to regrow. The idea of windowing is to smooth the sharp corners resulted from the clipping action. Here, a peak function is defined as

$$
p(n)=1-c(n)=1-\frac{A}{|x(n)|}
$$

where $p(n)$ is a real-valued function ranged from zero to one and can be regarded as the inverse function of $c(n)$. If $c(n)$ has lots of sharp corners, so has $p(n)$. (4) can be rewritten as

$$
c(n)=1-p(n)
$$

A simplest way to smooth the sharp corners in $p(n)$ of (5) is to filter it with a windowing function $w(n)$ to give a smooth version of the clipping signal, i.e., a new clipping function

$$
c^{\prime}(n)=1-p(n) * w(n)
$$

The window function used for study is the common window such as Hann, Hamming and Gaussian. For example, a Hann window and Gaussian window are given by

$$
\begin{gathered}
w(n)=0.5\left(1-\cos \left(2 \pi \frac{n}{L-1}\right)\right), \quad n=0, \ldots, L-1 \\
w(n)=\exp \left(-\frac{1}{2}\left(\lambda \frac{k-(L-1) / 2}{L / 2}\right)^{2}\right), \quad n=0, \ldots, L-1
\end{gathered}
$$

respectively where $L$ is the length of window. $\lambda$ in (8) is the reciprocal of the standard deviation. The number of data symbols spanned by the window length, $L$, determines the frequency bandwidth of the window.

The filtering process in (6) is the convolution of $p(n)$ and $w(n)$ and so the filtered signal could have envelop fluctuations with peak values greater than 1 . When this occurs, the new clipping function $c^{\prime}(n)$ in (6) will become negative and seriously degrade the EVM and the BER performance. Therefore, if it occurs in this technique, the negative values are replaced by zeros. (NB this is in fact also a clipping operation, i.e., the negative signals are clipped to zeros, causing the signal spectrum to regrow.) In (6), the new clipping function, $c^{\prime}(n)$, is then used to clip the signal, $x(n)$, according to (2), at the pulse shaping filter output to produce a new windowed signal in (9) but with reduced CF.

$$
y^{\prime}(n)=c^{\prime}(n) x(n)
$$

\subsection{Peak Windowing}

The windowing technique proposed here is similar to the conventional technique, but requires no convolution process. The signal envelope fluctuation resulted from using this technique is significantly less than those using the conventional technique. In this technique, the clipping function $c(n)$ and the peak function $p(n)$ as defined in (3) and (4), respectively, are obtained at the pulse shaping filter output in the same way as in the conventional windowing technique. In conventional windowing, each of the peaks in $p(n)$ is used as an impulse to produce a certain window. At each time instance, the resultant signal amplitude is the sum of all individual signal samples produced from that windows with the corresponding peaks in $p(n)$. However, in the proposed windowing technique, the signal value at each time instance is the maximum value among all the signal samples produced from the window functions with the corresponding peaks in $p(n)$. As a result, the resultant value at any instance will never exceed the peak value of $p(n)$ and so the values of $c^{\prime}(n)$ in (6) will never become negative. Thus the technique is expected to perform much better than the conventional windowing technique.

When windowing is applied to the signal, the important issues are the type of window and its length used. In (7) and (8), $L$ is the length of a window of any type and determines the number of data symbols spanned by the window. If $L$ is too large, then more data symbols will be affected, degrading the EVM and the BER. In contrast, if $L$ is too short, the frequency bandwidth will be too large to suppress the signal spectrum which regrows after the clipping process in (2). In this paper, an attempt is made to optimize the type, length, $L$, and some other parameters for different windows such as $\alpha$ of Gaussian window for the WCDMA system.

\section{SIMULATION RESULTS}

Computer-simulation tests using Matlab and Simulink have been carried out to study the effects of the conventional windowing and the peak-windowing technique on the signal quality metric, i.e. the EVM, the PCDE and the ACLR, and the $\mathrm{CF}$ and the BER performances of the system shown in Fig. 1. Initially, a Hann window given by (7) with a length of $L=73$, equivalent to 18 -symbol (or chip), was used in both techniques and their performances were compared. Results have showed that peak windowing is substantially better. Then extensive tests were used to optimize the different windows used in the two windowing techniques. 
Results have shown that Gaussian window performs the best. Moreover, a Gaussian window with $\lambda=2.5$ and length $L=25$, equivalent to 6 data symbol time, is optimum for conventional windowing. While a Gaussian window with $\lambda=5$ and length $L=81$, equivalent to 20 data symbol time, is optimum for peak-windowing in terms of CF reduction, yet satisfying the $3 \mathrm{GPP}$ requirements in [4]. Further discussion will be followed in the next section. In the 3GPP specification [4], the EVM and PCDE are defined as

$$
E V M=\sqrt{\frac{E\left[e^{2}\right]}{\alpha E\left[x^{2}\right]}} \text { and PCDE }=\max _{k}\left\{\frac{E\left[e_{k}^{2}\right]}{\alpha E\left[x^{2}\right]}\right\}
$$

where $e$ is the error signal, $x$ is the reference signal, $\alpha$ is a scaling factor chosen to minimize the EVM and PCDE by using least mean square method and $e_{k}$ is the error signal $e$ projected onto the spreading code $k$ in code domain and the set of $k$ used is defined in [4].

\subsection{Optimum Clipping Ratios}

In the 3GPP specification [4], the requirements for the signal quality on the EVM and the PCDE, are $17.5 \%$ and $33 \mathrm{~dB}$, respectively, and on the ACLR is $50 \mathrm{~dB}$ at $10 \mathrm{MHz}$. These values are used as the requirements for the WCDMA system studied. The effects of 1) the conventional windowing technique with Hann window length $L=73,2$ ) Gaussian window of $\lambda=2.5$ and length $L=25,3$ ) the peakwindowing technique with Hann window length $L=73$, and 4) Gaussian window of $\lambda=5$ and length $L=81$ on the EVM, the PCDE and the ACLR, with different CRs, are shown in Figs. 2a, 2b and 2c, respectively. Of course, the larger is the $\mathrm{CR}$ value, the less is the signal distortion, resulting in the smaller EVM and PCDE and higher ACLR values, at the expenses of the requirement to use a more linear PA and less efficient PA. However, from the operators' point of view, it is preferred to use a low cost system, i.e., using high efficient PAs, which means using a smaller CR value.

It can be seen from Figs. 2a, $\mathrm{b}$ and $\mathrm{c}$ that, in order to satisfy all requirements in terms of the EVM, the PCDE and the ACLR, for the case of conventional windowing using Hann window, the minimum CR values have to be 2.20 , 2.08 and 1.71, respectively. For the other three cases, the minimum $C R$ values are also simulated and shown in Table $\mathrm{I}$, where the bold numbers are the minimum $\mathrm{CR}$ values (or optimum $\mathrm{CR}$ values) required for the technique used to satisfy the $3 \mathrm{GPP}$ requirements and the numbers in brackets are the scaling factors given in (10).

\subsection{Reduction in $\mathrm{CF}$}

The unclipped WCDMA signal with 16 channels used in the study has the $\mathrm{CF}$ of $13.25 \mathrm{~dB}$. Computer simulation tests have been used to study the effects of the windowing techniques on the reduction of the CF for the WCDMA signal and

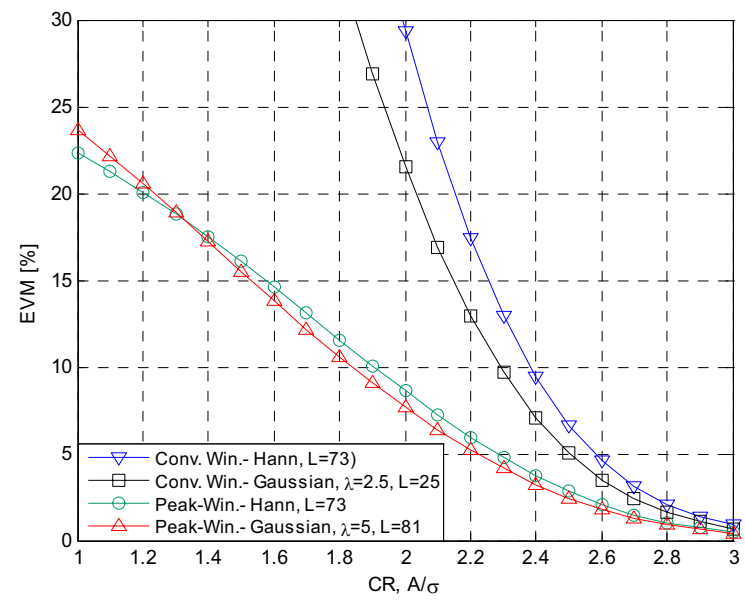

Figure 2a. EVM of signals using different techniques

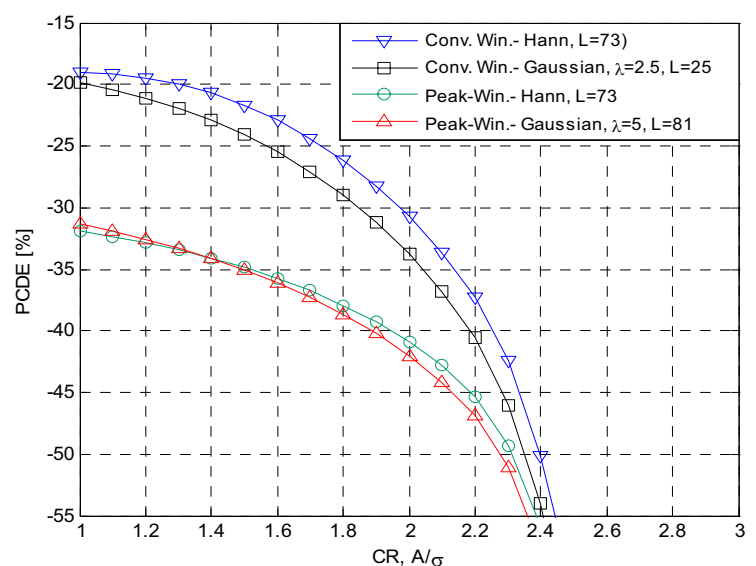

Figure $2 \mathrm{~b}$. PCDE of signals using different techniques

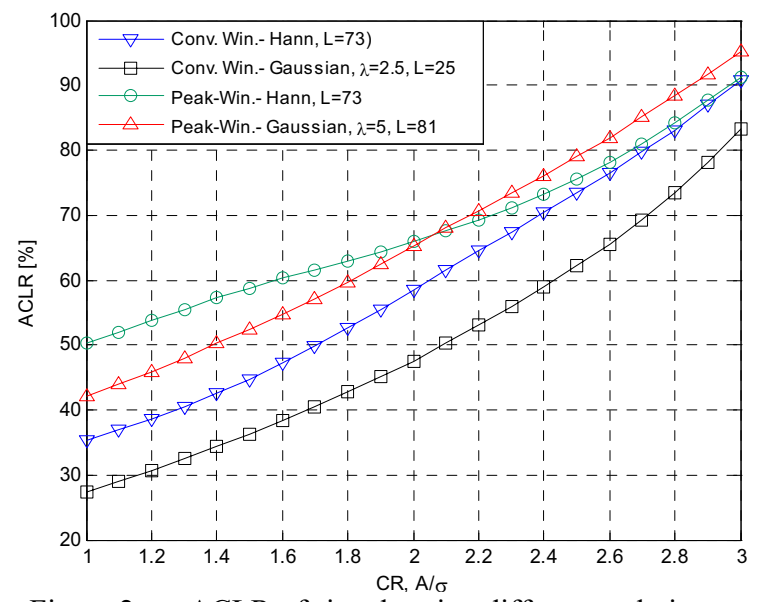

Figure 2c. ACLR of signals using different techniques

TABLE I. MAXIMUM CLIPPING RATIO LIMITED BY EVM, PCDE AND ACLR WITH THE CORRESPONDING OF $\alpha$

\begin{tabular}{|c|c|c|c|c|}
\hline & \multicolumn{4}{|c|}{ Windowing techniques } \\
\hline \multirow{2}{*}{ CR } & $\begin{array}{c}\text { Conv. Win. } \\
\text { Hann } \\
\mathrm{L}=73\end{array}$ & $\begin{array}{c}\text { Conv. Win. } \\
\text { Gaussian } \\
\mathrm{L}=25\end{array}$ & $\begin{array}{c}\text { Peak Win. } \\
\text { Hann } \\
\mathrm{L}=73\end{array}$ & $\begin{array}{c}\text { Peak Win. } \\
\text { Gaussian, } \\
\mathrm{L}=81\end{array}$ \\
\hline \multirow{2}{*}{ EVM } & $\mathbf{2 . 2 0}$ & $\mathbf{2 . 0 9}$ & $\mathbf{1 . 4 1}$ & 1.38 \\
& $(\alpha=0.94)$ & $(\alpha=0.95)$ & $(\alpha=0.87)$ & $(\alpha=0.83)$ \\
\hline PCDE & 2.08 & 1.97 & 1.25 & 1.26 \\
\hline ACLR & 1.71 & 2.09 & 1.04 & $\mathbf{1 . 3 9}$ \\
\hline
\end{tabular}


results are shown in Fig. 3, where $\Delta \mathrm{CF}=13.25 \mathrm{~dB}-$ new $\mathrm{CF}$ is the reduction of the $\mathrm{CF}$ or the difference in the $\mathrm{CF}$ with and without using the windowing technique. Of course, when a large $\mathrm{CR}, A / \sigma$, is used, the signal is clipped less severely. The differences in the $\mathrm{CF}$ with and without using the techniques are less significant and so the reduction in $\mathrm{CF}$ is smaller. From Fig. 3, the reduction of the $\mathrm{CF}$ in the WCDMA signal, using the conventional windowing and the peak-windowing with the corresponding optimum $\mathrm{CF}$ values shown in Table I, can be obtained and as shown in Table II.

In summary, before optimizing the window used in both techniques, $\triangle \mathrm{CF}$ of peak-windowing is $7.72 \mathrm{~dB}-5.53 \mathrm{~dB}=$ $2.19 \mathrm{~dB}$ more than conventional windowing. After optimizing the windows used in the two techniques, the optimum peak-windowing technique, i.e., with the use of Gaussian window with $\lambda=5$ and $L=81$ achieves $2.15 \mathrm{~dB}$ (i.e., $8.13 \mathrm{~dB}-5.98 \mathrm{~dB}$ ) more in reducing the $\mathrm{CF}$ than the optimized conventional windowing using Gaussian window with $\lambda=2.5$ and $L=25$.

\subsection{Bit-Error-Rate}

Computer-simulation results on the BER performance of the WCDMA system in Fig. 1, with the use of these techniques and the corresponding optimum CRs in Table II, are shown in Fig. 4. Obviously, the signal without using windowing has no signal distortion and performs the best. With the use of windowing, the signal is distorted by the non-linear processes, degrading the BER performance as can be seen in Fig. 4. All the four windowing techniques result in similar BER performances. However, the Fig. 4 does not give a fair comparison on the performances of the windowing techniques used because in these performances the $\mathrm{CF}$ reductions have not been taken into account. In these tests, the CR values used for the optimized conventional windowing and optimized peak windowing are 2.09 and 1.39, respectively, which produces the corresponding CF reductions as shown in Table II. As explained previously, these reductions in practice can be translated into the increases of the output powers from the PA, leading to higher SNRs. If this effect is taken into account, at $\mathrm{BER}=10^{-}$ 3 , the optimized peak windowing has about $8 \mathrm{~dB}$ gain in $\triangle \mathrm{CF}$ and $2 \mathrm{~dB}$ tradeoff in BER which gives a total $6 \mathrm{~dB}$, and $2 \mathrm{~dB}$ over the unclipped signal and the optimized conventional windowing respectively.

\section{CONCLUSIONS}

A peak-windowing technique has been proposed and then optimized to reduce the CF of the signal from the WCDMA base station and studied using computer simulation. Results show that peak windowing technique is superior to the conventional windowing technique both before and after the window optimization. It has been found that the optimum case of peak windowing is using Gaussian window with $\lambda=5$ and 20 data symbol time of length. With the use of the above window, results have shown that, at $\mathrm{BER}=10^{-3}$, the technique has an advantages of $6 \mathrm{~dB}$ and $2 \mathrm{~dB}$ over the unclipped signal and conventional windowing technique.

TABLE II. REDUCTION IN CF BY DIFFERENT TECHNIQUES

\begin{tabular}{|c|c|c|c|c|}
\hline & \multicolumn{4}{|c|}{ Windowing techniques } \\
\hline & $\begin{array}{c}\text { Conv. Win. } \\
\text { Hann } \\
\text { L=73 }\end{array}$ & $\begin{array}{c}\text { Conv. Win. } \\
\text { Gaussian } \\
\text { L=25 }\end{array}$ & $\begin{array}{c}\text { Peak Win. } \\
\text { Hann } \\
\text { L=73 }\end{array}$ & $\begin{array}{c}\text { Peak Win. } \\
\text { Gaussian } \\
\text { L=81 }\end{array}$ \\
\hline Min. CR & 2.20 & 2.09 & 1.41 & 1.39 \\
\hline$\Delta$ CF $[\mathrm{dB}]$ & $\mathbf{5 . 5 3}$ & $\mathbf{5 . 9 8}$ & $\mathbf{7 . 7 2}$ & $\mathbf{8 . 1 3}$ \\
\hline
\end{tabular}

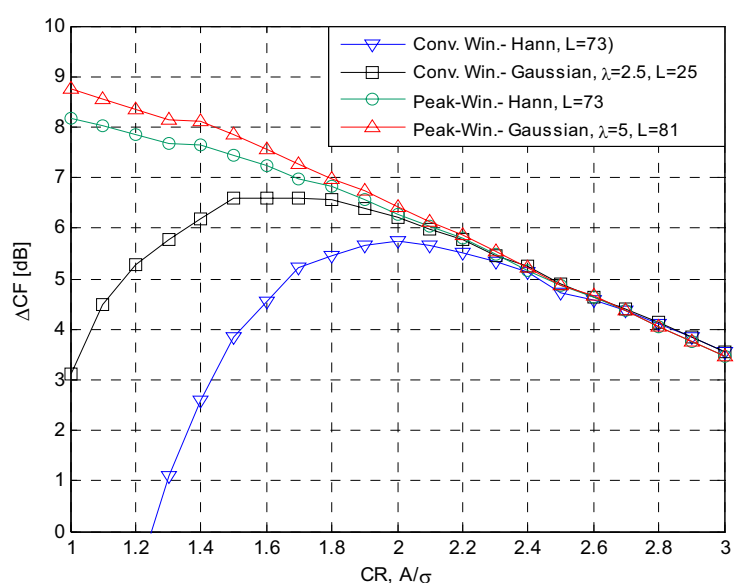

Figure 3. Reduction in $\mathrm{CF}$ of signals using different techniques

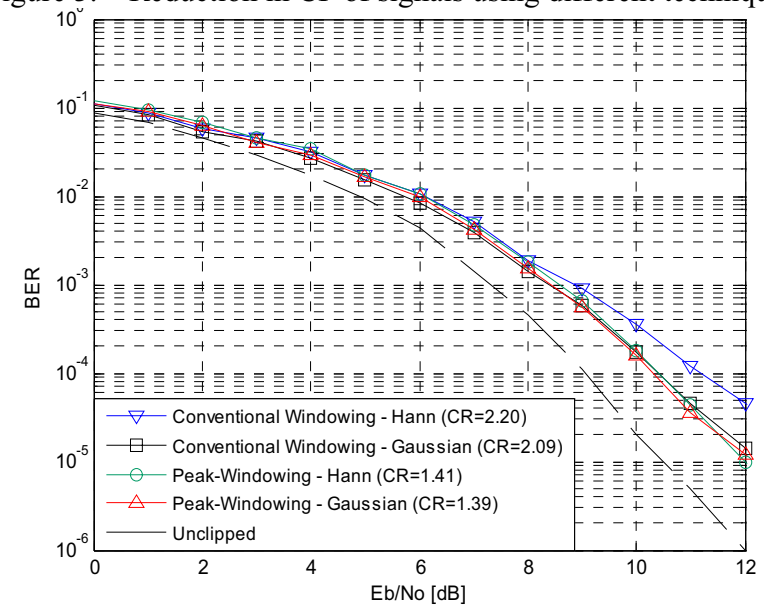

Figure 4. BER performance of signals using different techniques

\section{REFERENCES}

[1] S. Boyd, "Multitone Signals with Low Crest Factor," IEEE Trans. on Circuits and Systems, vol. cas-33, Oct. 1986, pp. 1018-1022.

[2] X. Li and L. J. Cimini, Jr., "Effects of clipping and filtering on the performance of OFDM," in Proc. VTC'97, May 1997, pp. 1634-1638.

[3] Richard van Nee and Arnout de Wild, "Reducing the Peak-toAverage Power Ratio of OFDM", IEEE Vehicular Technology Conference, 1998 vol. 3, pp. 2072-2076.

[4] 3GPP Technical Specification Group Access Network Base station conformance testing, TS 25.141 V6.7.0, 2004. 